\title{
OPTOACOUSTIC DETECTION OF MICROPARTICLES IN LIQUIDS AT LASER FLUENCES BELOW THE OPTICAL BREAKDOWN THRESHOLD
}

\author{
S. S. ALIMPIEV and Ya. O. SIMANOVSKII \\ General Physics Institute, Russian Academy of Sciences \\ ul. Vavilova 38, Moscow 117942, Russia \\ S. V. EGEREV and A. E. PASHIN \\ N. N. Andreev Acoustics Institute \\ ul. Shvernika 4, Moscow 117036, Russia
}

(Received 16 March, 1994)

\begin{abstract}
An optoacoustic device consisting of a $\mathrm{XeCl}$ excimer laser and a measurement cell with an attached piezotransducer was used for detecting microparticles suspended in liquid probes. The potential of optoacoustic diagnostics of microinhomogeneous liquids was enhanced by applying informative parameters of optoacoustic response. Probes of distilled water and Dow Chemical latex suspension were used in experiments. It was shown that cavitation mechanism of optoacoustic conversion taking place at laser fluences below the optical breakdown threshold was well-suited for individual microparticle detection. The approach proposed is different from the well-known breakdown counting optoacoustic technique.
\end{abstract}

KEY WORDS: Microinhomogeneous liquids, laser-induced cavitation.

\section{INTRODUCTION}

Optoacoustic conversion consists in inducing acoustic waves in a liquid sample by its irradiation with a light beam of varying intensity. The phenomenon is used as a basis of the well-established version of liquid spectroscopy. ${ }^{1}$

The mechanisms of laser sound generation are various and their relative contributions depend strongly on the released energy density. At a small energy deposit, the linear thermooptical effect dominates in the process of sound generation. This effect is based on the thermal expansion of the portion of the media where the light was absorbed. The magnitude of acoustic response appears to be proportional to the optical absorbance of a liquid. This relation forms the basis of low-energy optoacoustic spectroscopy of homogeneous liquids and related tasks. The presence of lightabsorbing microparticles (dust or colloidal particles, for example) in a liquid probe 
changes essentially the pattern of sound generation due to "delayed" heat deposit from particles to liquid. ${ }^{2}$ The efficiency of the process increases if the energy input exceeds the threshold of vaporization of the liquid surrounding the absorbing particles. As a result of liquid vaporization bubbles appear, and their expansion leads to effective sound generation. ${ }^{3}$ At higher energy deposits, the light-absorbing particles in the fluid play the role as nuclei of optical breakdown of a liquid. ${ }^{4}$ The further increase of the light intensity obtained, for example, by focusing of the laser beam leads to the formation of laser spark developing in the focal region. ${ }^{5}$

The effect of microparticles' disturbing contribution has been observed in a number of experimental studies in optoacoustic spectroscopy, ${ }^{6-8}$ statistical properties of optoacoustic signal being of special interest. At the same time, optoacoustic conversion can be an efficient tool for assaying microparticles. The $\mathrm{CW}$ optoacoustic conversion was used to detect the presence of relatively large particles of $\mathrm{BaSO}_{4}$ (of about $10 \mu \mathrm{m}$ and larger) in water. ${ }^{9}$ Unfortunately, the use of $\mathrm{CW}$ optoacoustic methods has the disadvantage of low sensitivity. The optoacoustic contribution of individual particles immersed into non-absorbing liquid have been studied theoretically in the case of pulsed laser excitation. ${ }^{10}$

Papers ${ }^{11-14}$ treat the possibility of microparticles detection by using the laser breakdown mechanism of optoacoustic conversion. Statistical properties of optoacoustic response have been applied to detect submicron polystyrene particles at ultra-low concentration. The disadvantage of the developed technique is that a very high intensity of the laser radiation is required resulting in a very small detection volume.

The subject of the present study is the development of below-threshold-fluence optoacoustic diagnostics of diluted suspensions. Fluences are limited so that there is no laser spark formation. Vapor bubble formation is instead observed as a set of individual events, and the possibility of a single particle detection is thus provided.

\section{THEORY}

During optoacoustic studies of homogeneous solutions one is faced (as a rule) with only one informative parameter of the acoustic response, i.e. the pulse magnitude $p$ which can be expressed for a cylindrical laser beam of radius a in the following form: ${ }^{1}$

$$
\mathrm{p} \approx\left(\alpha \beta \mathrm{u}^{2} \mathrm{E}\right) /\left(\pi \mathrm{a}^{3 / 2} \mathrm{c}_{\mathrm{p}} \mathrm{r}^{1 / 2}\right),
$$

where $\mathrm{E}$ is the laser pulse energy, $\mathrm{r}$ is the distance of the observation point from the beam axis, $\alpha$ is the absorption coefficient of the probe, $u, \beta$ and $c_{p}$ are velocity of sound in liquid, isobaric volume expansion and heat capacity coefficients. However the applicability of the expression (1) is restricted to the cases when it is possible to neglect contributions due to microparticle-induced additional conversion mechanisms. In the case under study one also have to consider other informative parameters, such as statistical parameters, energy dependence of averaged response magnitude, time domain and frequency domain parameters of acoustic response. 


\section{Statistical Parameters}

One can represent the results of a series of acoustic response magnitude measurements in the form of a histogram. By analyzing the form of the histogram and its approximation by means of some known probability distribution law, it is possible to estimate a sample's content. It is known that in the case of a homogeneous liquid, the sample thermoelastic response is of good repeatability. The resulting histogram can be approximated with good accuracy by the Gauss probability distribution law; the mean magnitude value $\left\langle p>\right.$ coincides with (1), also the standard deviation, $\sigma_{\mathrm{p}}$ is defined by the level of the reception system noise.

At low laser fluences, the additional response of the liquid due to the thermal relaxation of .microparticle system within the mono-dispersion approximation is described by the following mean magnitude $\left\langle\mathrm{p}_{\mathrm{par}}\right\rangle::^{15}$

$$
<\mathrm{p}_{\text {part }}>=\left(\beta \mathrm{Nk}_{\mathrm{a}} \mathrm{E} / \mathrm{c}_{\mathrm{p}} \mathrm{R}\right)\left(\pi \mathrm{u} \chi^{3 / 8 \mathrm{r}}\right)^{1 / 2},
$$

where $x$ is the thermal diffusivity of a particle, $\mathrm{N}$ is the number concentration of particles, $\mathrm{R}$ is their radius and $\mathrm{k}_{\mathrm{a}}$ is the dimensionless absorption efficiency of particle absorption which depends on optical constants of the particle material as well as on the laser wavelength $\lambda$ to $R$ ratio. For dielectric particles having refraction index $n$

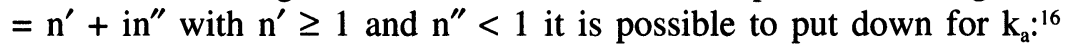

$$
\mathrm{k}_{\mathrm{a}}=\left[1-\exp \left(-8 \pi \mathrm{n}^{\prime \prime} \mathrm{R} / \lambda\right)\right] \cdot \exp \left[-0.2\left(\left(\mathrm{n}^{\prime 2}-\mathrm{n}^{\prime \prime 2}\right)^{1 / 2}-1\right)\right] \text {. }
$$

It follows from (2) that the ratio of standard deviation $\sigma_{\mathrm{p}}$ of the low-energy particle contribution to the mean magnitude can be put down as $\sigma_{\mathrm{p}} /\left\langle\mathrm{p}_{\text {part }}\right\rangle \approx\left(\mathrm{V}_{\mathrm{O}} \mathrm{N}\right)^{-1 / 2}$ where $\mathrm{V}_{\mathrm{O}}$ is the volume of the laser-induced acoustic source (the intersection of the laser beam and the sample). It is possible to detect the component (2) of acoustic response, caused by the particles, if $\mathrm{N}$ is not too small, hence the inequality $\sigma_{\mathrm{p}} /\left\langle\mathrm{p}_{\mathrm{part}}\right\rangle \ll 1$ is valid. As a result, one is faced with good repeatability of the total response at low energy deposits.

At a higher laser fluence, interaction of the laser beam with a weakly absorbing inhomogeneous liquid can lead to strong heating of immersed particles. Their temperature can exceed the boiling temperature of the host liquid. It is possible to roughly estimate the corresponding fluence threshold value $\varepsilon_{\mathrm{th}}$ for a single absorbing particle as follows:

$$
\varepsilon_{\mathrm{th}}=\left(4 \mathrm{R} / \mathrm{k}_{\mathrm{a}}\right) \Delta \mathrm{T}(\rho \mathrm{c})_{\text {particle }}
$$

where $\Delta \mathrm{T}$ is the difference between the boiling point and the initial temperature of the liquid. For small particles $\left(8 \pi n^{\prime \prime} R / \lambda \ll 1\right) k_{a}$ is proportional to $R$, and the value $\varepsilon_{\mathrm{th}}$ is independent of their radii. For sufficiently large or strongly absorbing particles $\left(8 \pi n^{\prime \prime} \mathrm{R} / \lambda \gg 1\right), \mathrm{k}_{\mathrm{a}} \approx 1$ is valid, and the value $\varepsilon_{\mathrm{th}}$ increases with the particle radius. Let us estimate the threshold value $\varepsilon_{\mathrm{th}}$ for typical atmosphere aerosol microparticles ${ }^{17}$ which are most often found in water, assuming $\Delta \mathrm{T}=80^{\circ}$. For strongly absorbing carbon particles $\left(\mathrm{k}_{\mathrm{a}} \approx 1\right)$, the corresponding values of the fluence threshold $\varepsilon_{\mathrm{th}}$ can be estimated as $2 \cdot 10^{-3} \mathrm{~J} / \mathrm{cm}^{2}$ (for $\mathrm{R}=0.1 \mu \mathrm{m}$ ), $2 \cdot 10^{-2} \mathrm{~J} / \mathrm{cm}^{2}$ (for $\mathrm{R}=1 \mu \mathrm{m}$ ), and $0.2 \mathrm{~J} / \mathrm{cm}^{2}$ (for $\mathrm{R}=10 \mu \mathrm{m}$ ). For weakly absorbing dust microparticles (quartz ones, 
for example) having $\mathrm{n}^{\prime \prime}=0.06-0.025, \mathrm{R}=0.1 \mu \mathrm{m}$ and $\mathrm{k}_{\mathrm{a}} \approx 0.9$ the estimation of $\varepsilon_{\mathrm{th}}$ gives a value of $(2-5) \cdot 10^{-3} \mathrm{~J} / \mathrm{cm}^{2}$. In the case of high quality polystyrene latex, $\varepsilon_{\mathrm{th}}$ can be estimated as $10^{-1} \mathrm{~J} / \mathrm{cm}^{2}$.

To analyze the peak pressure response of the near-focal point volume as a function of laser energy we will use the simple model assuming the spherical symmetry of expanding vapor bubbles and taking into account their collective response. The elementary expanding vapor cavity is an effective radiator of a spherical sound wave. Cavitation mechanism "switches on" in that very region of the beam where the following threshold condition is valid:

$$
\varepsilon(\mathrm{x}, \mathrm{y}, \mathrm{z})>\varepsilon_{\mathrm{th}},
$$

where the function $\varepsilon(\mathrm{x}, \mathrm{y}, \mathrm{z})$ describes the energy density distribution within the laser beam. If there are only absorbing particles of equal radii $R$ and if the vapor in the bubble is transparent to incident light, we can put the energy balance equation for a single expanding cavity with coordinates $(\mathrm{x}, \mathrm{y}, \mathrm{z})$ at the stage of energy deposit as follows:

$$
\left(\mathrm{h} \rho_{\mathrm{v}} / \mathrm{k}_{\mathrm{a}} \pi \mathrm{R}^{2}\right)(\partial \mathrm{V}(\mathrm{t}) / \partial \mathrm{t})=\partial\left[\varepsilon(\mathrm{x}, \mathrm{y}, \mathrm{z}) \mathrm{g}(\mathrm{t})-\varepsilon_{\mathrm{th}}\right] / \partial \mathrm{t}, \quad \mathrm{t}<\tau,
$$

where $\mathrm{h}$ is the enthalpy difference between the initial water stage and its critical point, $\rho_{\mathrm{v}}$ is the vapor density, $\mathrm{V}(\mathrm{t})$ is the current bubble volume, $\tau$ is the laser pulse width, and $\mathrm{g}(\mathrm{t})$ is the temporal pulse profile. It is possible to take parameters of the critical point (for water $\mathrm{h}=3000 \mathrm{~J} / \mathrm{g}, \rho_{\mathrm{v}}=0.3 \mathrm{~g} / \mathrm{cm}^{3}$ ) and write $\mathrm{V}(\tau)$ as $\mathrm{V}(\tau) \cong$ $\tau[\partial \mathrm{V}(\mathrm{t}) / \partial \mathrm{t}]_{\mathrm{t}=0}$. For the leading spike of the compression pulse radiated by the bubble during the energy deposit stage we can use the following expression: ${ }^{18}$

$$
\mathrm{p}\left(\mathrm{t}-\frac{\mathrm{r}}{\mathrm{u}}\right)=\rho \frac{3 \mathrm{~V}(\tau)}{4 \pi \tau^{2} \mathrm{r}} \quad \frac{\mathrm{t}-(\mathrm{r} / \mathrm{u})}{\tau}, \quad \mathrm{t}-(\mathrm{r} / \mathrm{u}) \leq \tau,
$$

where $\rho$ is the liquid density. As follows from (7), the peak pressure of the pulse occurs at the moment $\mathrm{t}-(\mathrm{r} / \mathrm{u})=\tau$. During a long period after the laser pulse is over, the bubble is characterized by much slower expansion. The work is done against the outer pressure in the liquid, and the kinetic energy is transformed into potential energy stored in the expanded bubble. The phase of rarefaction is emitted during this stage. From the analysis of the previous expressions it is evident that for single particle the linear dependence of the peak pressure $p_{\text {bubl }}$ with respect to the energy deposit is expected:

$$
\mathrm{p}_{\text {bubl }}=\left(3 / 4 \pi \mathrm{r} \rho_{\mathrm{v}} \mathrm{h}\right) \rho \mathrm{k}_{\mathrm{a}} \mathrm{R}^{2}\left[\varepsilon(\mathrm{x}, \mathrm{y}, \mathrm{z})-\varepsilon_{\mathrm{th}}\right] .
$$

It follows from (8) that the considered mechanism is characterized by a significant contribution of individual particles satisfying the threshold condition (5). In the case of monodisperse suspension it is easy to detect the phenomenon by studying the resulting histograms which suffer broadening thus giving $\left(\sigma_{\mathrm{p}} /<\mathrm{p}_{\text {bubl }}>\right) \approx 1$. In the case of polydisperse suspension it is natural to expect more complicated, nonGaussian types of response-magnitude probability distribution. 


\section{Energy Dependence of Averaged Response Magnitude}

The linear segment of $<p>$ (E) dependence of the type (1), (2) gives way to non-linear types as energy grows up. The corresponding exponents additionally characterize the type of suspension. Let us again turn to the model of a diluted monodisperse suspension. By taking into account the simultaneous radiation of sound by numerous bubbles satisfying the threshold condition we can explain the non-linear growth of the averaged peak pressure $<p>$ with E. Using (7), (8) we obtain in the far field in the direction perpendicular to the laser beam axis

$$
<\mathrm{p}>=\left(3 / 4 \tau_{1}^{2} \mathrm{r} \rho_{\mathrm{v}} \mathrm{h}\right) \mathrm{N} \rho \mathrm{k}_{\mathrm{a}} \mathrm{R}^{2_{\mathrm{o}}} \int_{\mathrm{V}_{\mathrm{o}}} \mathrm{dV} \mathrm{o}\left(\varepsilon(\mathrm{x}, \mathrm{y}, \mathrm{z})-\varepsilon_{\mathrm{th}}\right),
$$

where the coordinates of the volume $V_{0}$ satisfy (5). Assuming uniform energy density distribution in the laser beam cross-section and using the expression for $\varepsilon(x, y, z)$ in the form $\varepsilon(\mathrm{x}, \mathrm{y}, \mathrm{z}) / \varepsilon_{0}=a_{0}^{2} /\left[a_{0}^{2}+(\mathrm{z} / \mathrm{m})^{2}\right]$, where $\mathrm{a}_{0}$ is the focal spot radius, $\mathrm{m}=$ $2 \pi \mathrm{a}_{0} / \lambda, \varepsilon_{0}$ is the maximum energy density in the focal spot, we finally obtain

$$
<\mathrm{p}_{\text {bulb }}>=\left(1 / 2 \tau^{2} \mathrm{r} \rho_{\mathrm{v}} \mathrm{h}\right) \mathrm{N} \rho \mathrm{k}_{\mathrm{p}} \mathrm{R}^{2} \mathrm{a}_{0}^{3} \varepsilon_{\mathrm{th}} \mathrm{m}\left[\left(\mathrm{E} / \pi \mathrm{a}_{0}^{2} \varepsilon_{\mathrm{th}}\right)-1\right]^{3 / 2} .
$$

It should be mentioned that similar calculations with the Gaussian beam profile gives the same result. If there are particles of different size immersed in the host liquid, a stronger than of power $3 / 2$ dependence $\langle p\rangle(E)$ is expected. Here we are to take into account the concrete form of $n(R)=d N / d R$ and the energy threshold condition for $\varepsilon_{\mathrm{th}}$ which now depends on the particle size. It has been shown ${ }^{3}$ that a uniform law of carbon particle size distribution provides the dependence $<p>\alpha E^{4}$.

Thus, the laser-induced buble formation, accompanied by the intense sound generation due to the expansion of the buble, results in additional acoustic response of an inhomogenious liquid sample irradiated with a pulsed laser beam. The laser fluence threshold of buble formation strongly depends on the size as well as the optical and thermal constants of the particle material. As a consequence the acoustic response undergoes sufficient fluctuations depending on the particle properties and its position within the irradiated volume. The effect may find application in detection of individual microparticles in high purity liquids. Analysis of the acoustic signal statistics can be used to estimate the content of the liquid sample. Moreover, the additional information on the optical properties and size distribution of microparticles can be obtained by analyzing the laser fluence dependence of the averaged acoustic response magnitude.

\section{EXPERIMENTAL}

The experimental studies were conducted with the aid of the setup presented in Figure 1. The pulse of a XeCl excimer laser $\left(\lambda=308 \mathrm{~nm}\right.$ and $\left.\tau_{1}=30 \mathrm{~ns}\right)$ passed through a diaphragm, an attenuator and a quartz lens and then was focused into an optoacoustic measurement cell filled with the analyzed suspension probe. The cell of stainless steel having quartz windows is analogous to that described in Ref.' A piezoelectric transducer having the responsivity $7 \mu \mathrm{V} / \mathrm{Pa}$ and the frequency band up 


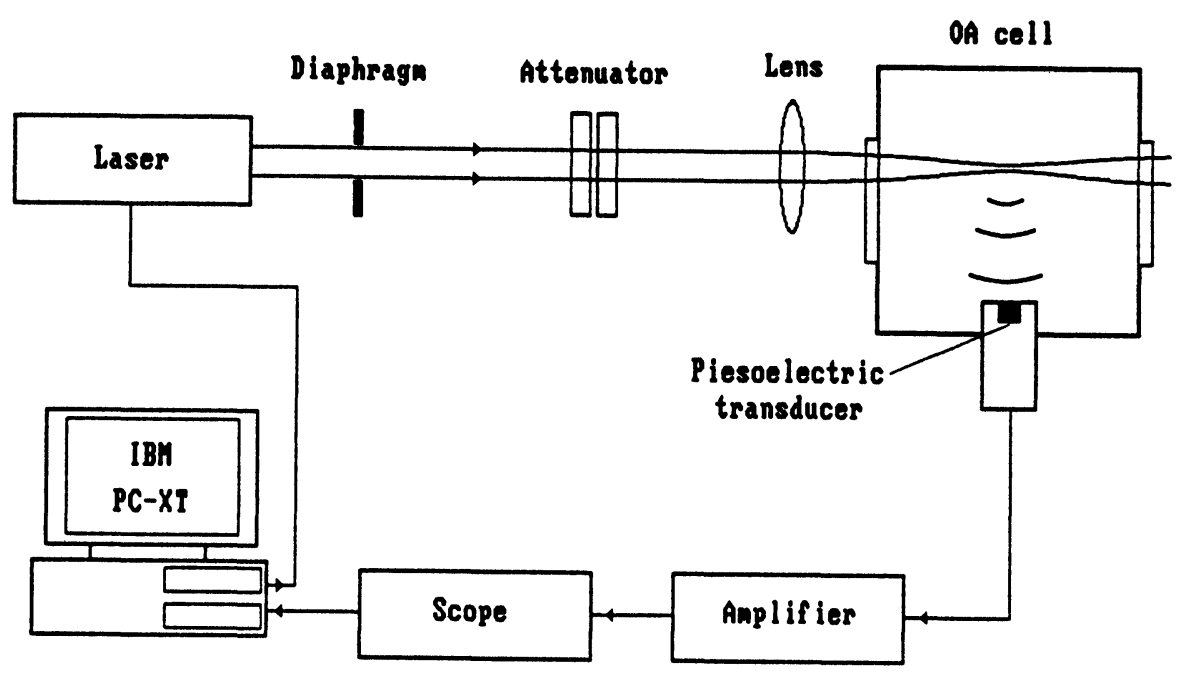

Figure 1 Experimental setup.

to $200 \mathrm{kHz}$ was attached to the cell. The cell was filled with a filtered distilled water probe containing particles with radii less then $0.3 \mu \mathrm{m}$. Other probes represented unfiltered water and diluted Dow Chemical latex suspension $(\mathrm{R}=2.2 \mu \mathrm{m})$.

The output signal of the transducer passed through the preamplifier with the gain $\mathrm{K}_{\mathrm{u}}=32$. After additional amplification by the final stage amplifier, the signal was recorded by a digitizing oscilloscope (S9-8, sampling frequency $20 \mathrm{MHz}$ ) and transferred to an IBM PC-XT computer. The laser energy measurements were provided by means of a calorimeter. The original software was used for signal series (1000 shots) recording and histogram analyzing. The averaged series magnitude and standard deviation were calculated at this very stage.

\section{RESULTS AND DISCUSSION}

The experimental histograms of acoustic response of two irradiated probes are presented in Figure 2. The first probe represents double distilled unfiltered water, while the second one represents filtered water with particles larger than $0.3 \mu \mathrm{m}$ removed. The three pairs of histograms are associated with three different laser fluences. Microparticles exhibit themselves by varying the probability behavior of the response as the energy level grows up. At the below-threshold level both histograms are of symmetrical type (Figure $2 \mathrm{a}$ ). Their width is defined by laser energy fluctuations and the transducer noise level. The shift of the most probable magnitude value of the unfiltered water with respect to the filtered one corresponds to particles contribution (2). This contribution is of linear thermal relaxation origin. No cavitation contribution can be found at this stage. 

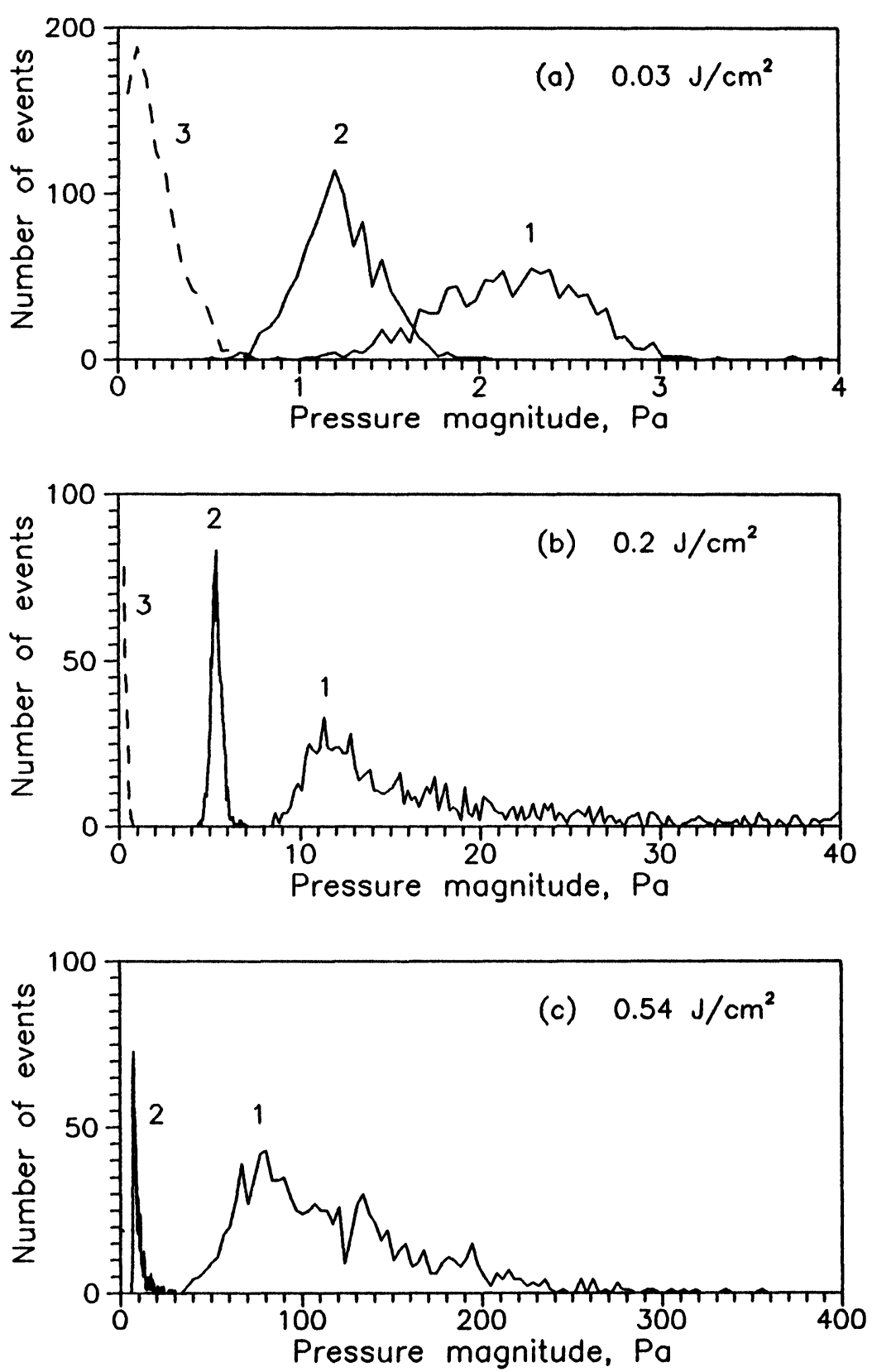

Figure 2 Histograms of acoustic pressure magnitude for unfiltered (1) and filtered (2) distilled water at different laser fluences. Dashed curve (3) represents the noise signal. 
The second pair of histograms (Figure $2 \mathrm{~b}$ ) corresponds to a fluence of $0.19 \mathrm{~J} / \mathrm{cm}^{2}$ which is well above the cavitation threshold. The comparison of two curves shows that smáll particles still don't change the histogram type, while the presence of large particles leads to the formation of a "long-tailed" histogram. The latter distribution is of a sufficiently larger standard deviation as well, which is due to a small amount of the "above-threshold" particles in the conversion region. Notice, that this circumstance is consistent with the consideration of the previous theoretical section. It should be said that the most probable magnitude value still corresponds to the linear thermal relaxation contribution. This intermediate energy regime is the optimal one for individual microparticles detection.

And, finally, the histograms of Figure $2 \mathrm{c}$ illustrate the case of a sufficiently large energy deposit of $0.54 \mathrm{~J} / \mathrm{cm}^{2}$ when the cavitation mechanism dominates for unfiltered water and becomes significant for filtered probe containing microparticles with radii under $0.15 \mu \mathrm{m}$.

It is useful to compare the above discussed histograms to the fluence dependence of the averaged response magnitude. Such dependence is presented in Figure 3 and corresponds to that very energy interval where one can follow the transition from the thermal relaxation mechanism of sound generation to the cavitation one. The dependence $\langle p\rangle(E)$ is of linear type if the fluence is under $0.2 \mathrm{~J} / \mathrm{cm}^{2}$. The nonlinear dependence observed at higher fluences is well approximated by $\langle\mathrm{p}\rangle \alpha \mathrm{E}^{2}$ if $\left.\varepsilon_{\mathrm{m}}\right\rangle$ $0.5 \mathrm{~J} / \mathrm{cm}^{2}$. The reception in the intermediate fluence range $\left(0.2-0.5 \mathrm{~J} / \mathrm{cm}^{2}\right)$ is, probably, influenced by the steepening of the front spike of the response which results in high frequency resonance excitation of the transducer.

The histograms of Figure 4 were obtained for the mixture of diluted latex suspension and the initial particles content of the filtered distilled water used as a host liquid. The latex concentration comprised $1.9 \times 10^{5} \mathrm{~cm}^{-3}$. This amount was sufficient to distort the left edge of the initial histogram. The growth of the averaged magnitude was another distinguishing feature. It was found in accordance with theoretical presumptions that the monodisperse component (latex) changes the left edge of the histogram while the polydisperse component (initial particles) holds on the right edge. This feature becomes evident if one plots histograms in log-log coordinates (Figure 4b). Thus by analyzing the histograms of acoustic response one can distinguish between polydisperse and monodisperse suspensions.

By comparing the histograms in Figure 2 (b) and (c) one finds that the larger particles present in unfiltered water can be detected at the lower laser fluence, even though in accordance with the above theoretical estimations the threshold of bubble formation increases with the particle radius. The reason is that in both cases the laser fluence is well above the cavitation threshold and the ratio between acoustic response magnitude and noise level is of critical importance. This is evident from the following rough estimate of the detection threshold for a single quartz particle of $0.1 \mu \mathrm{m}$ radius in water. For the typical piezotransducer responsivity $20 \mu \mathrm{V} / \mathrm{Pa}$ and amplifier spectral noise density $2 \mathrm{nV} / \mathrm{Hz}^{1 / 2}$ (in the frequency band $0.01-1 \mathrm{MHz}$ ) the corresponding rms noise level comprises $0.1 \mathrm{~Pa}$. For the given values it follows from (10) that the signal-to-noise ratio of about 3 is obtained if $E / \pi a_{0}^{2}>0.02 \mathrm{~J} / \mathrm{cm}^{2}$. In practical cases 


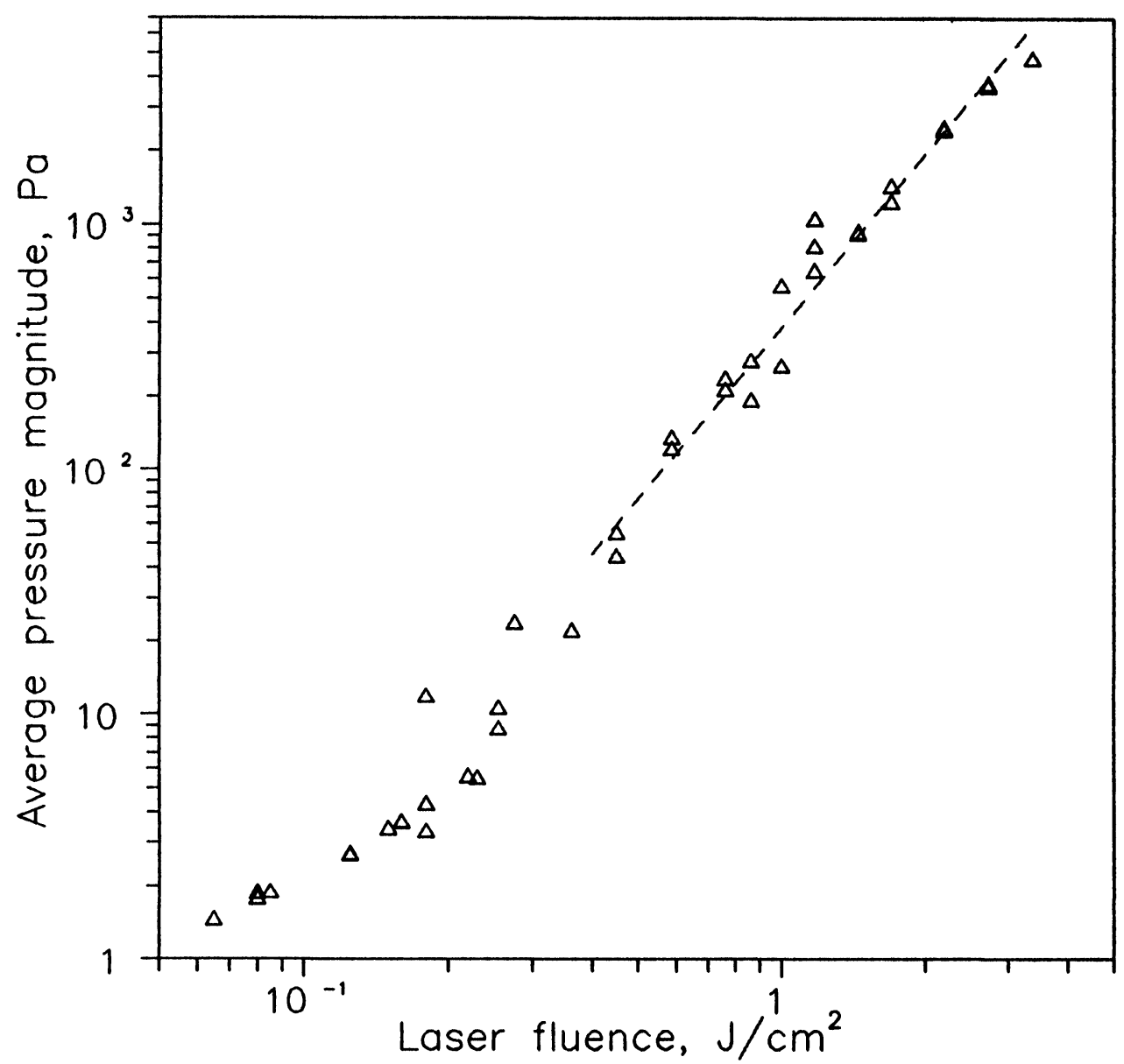

Figure 3 Plot of mean pressure magnitude versus the laser fluence for filtered distilled water. The best fit line (dashed line) has a slope of 2.33 .

the intensity of the ambient acoustic noise may range up to ten times of that of the amplifier, and it is necessary to have the laser fluence over $0.2 \mathrm{~J} / \mathrm{cm}^{2}$ to obtain the same signal-to-noise ratio.

The experimental results suggest that for a $\mathrm{XeCl}$ laser radiation the fluence of $0.5 \mathrm{~J} / \mathrm{cm}^{2}$ in the detection volume is sufficient for counting atmospheric microparticles with radii under $0.15 \mu \mathrm{m}$ suspended in water. The value is well below the threshold of optical breakdown observed at the laser fluence of about $20 \mathrm{~J} / \mathrm{cm}^{2}$. As a result, at a fixed laser energy the detection volume and hence the efficiency of the particle counting is two-three orders greater than that available in the laser breakdown method."

Individual microparticles counting provides a very low detection limit for insoluble impurities in water. Thus for quartz microparticles of $0.1 \mu \mathrm{m}$ radius and $10^{2} \mathrm{~cm}^{-3}$ concentration it can be estimated as 1 ppt. Furthermore, the use of laser sources with 

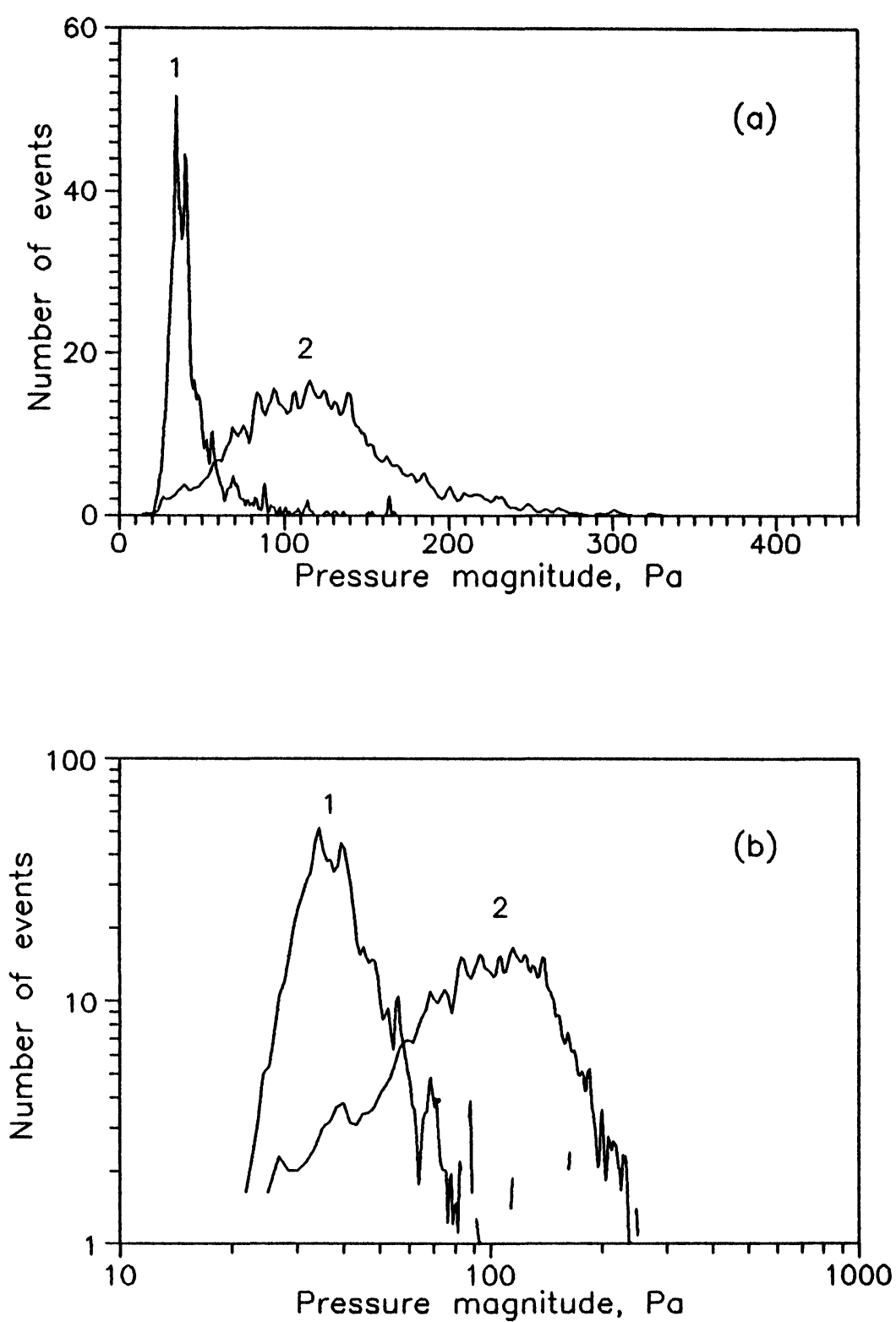

Figure 4 Histograms of acoustic pressure magnitude for filtered distilled water (1) and the same water with addition of Dow latex $(2.2 \mu \mathrm{m})$ suspension (2) in linear (a) and $\log -\log (\mathrm{b})$ coordinates.

different wavelengths makes it possible to analyze suspensions of microparticles varying in composition. In our experiments with an excimer pumped dye laser operating in the visible region (red and green) no bubble formation took place up to the laser-induced breakdown in the suspension of quartz particles weakly absorbing the 
laser radiation, whereas in the suspension of strongly absorbing carbon particles this process was observed under $1.06-\mu \mathrm{m}$ laser irradiation. ${ }^{3}$

\section{CONCLUSION}

It has been shown that the optoacoustic conversion in microinhomogeneous liquids exhibits itself mainly by means of light absorption by microparticles and subsequent processes, i.e. thermal relaxation and cavitation. There exists individual energy threshold of bubble formation depending on the particle material and radius. The below-threshold energy regimes are optimal to apply laser induced cavitation for individual particles detection. The informative parameters of acoustic response involve statistical parameters, energy dependence of averaged response magnitude and time domain and frequency domain parameters.

$\mathrm{A} \mathrm{XeCl}(\lambda=308 \mathrm{~nm})$ excimer laser was used to detect atmospherical microparticles as well as artificial latex suspension. The individual particles detection regime provides the responsivity level of the method of about $1 \mathrm{ppt}$.

\section{Acknowledgements}

This work was supported, in part, by a Soros Foundation Grant awarded by the American Physical Society. Support of the experimental program was from the Ministry of Science and Technical Policy, Russia, under Grant No. 801F.

\section{References}

1. C. K. N. Patel and A. C. Tam, Rev. Mod. Phys., 53, 517 (1981).

2. T. Kitamori, M. Fuji, T. Sawada and Y. Gohshi, J. Appl. Phys., 58, 1456 (1985).

3. P. I. Golubnichii, G. S. Kalyuzhnii and S. D. Korchikov, Zhurnal Prikladnoi Matematiki $i$ Tekhicheskoi Fiziki, No. 1 (143), 9 (1984) (in Russian).

4. W. Lauterborn, Acustica, 31, No. 2, 51 (1974).

5. P. A. Barnes and K. E. Rieckhoff, Apll. Phys. Lett., 13, 282 (1968).

6. J. I. Kim, R. Stumpe and R. Klenze, In: Topics in Current Chemistry, Vol. 157, (Springer-Verlag, Berlin, Heidelberg et al., 1990), pp. 129-183.

7. O. A. Roveri, G. M. Bilmes, K. Heihoff and S. E. Braslavsky, Appl. Spectroscopy, 44, 1706 (1990).

8 J. V. Beitz, M. M. Doxtader, V. A. Maroni, S. Okjima and D. T. Reed, Rev. Sci. Instrum., 61, 1395 (1990).

9. T. Savada and T. Kitamori, In: Physical acoustics, edited by W. P. Mason and R. N. Thurston, Vol. XVIII, (Academic Press, Boston et al., 1988), pp. 348-402.

10. G. J. Diebold and P. J. Westervelt, J. Acoust. Soc. Amer., 84, 2245 (1988).

11. T. Kitamori, K. Yokose, K. Suzuki, T. Sawada and Y. Gohshi, Japan. J. Appl. Phys., 27, 1983 (1988).

12. T. Kitamori, K. Yokose, M. Sakagami and T. Sawada, Japan. J. Appl. Phys., 28, 1195 (1989).

13. H. Fujimori, T. Matsui, T. Ajiro, K. Yokose, Hsueh Yu-Ming and S. Izumi, Japan. J. Appl. Phys., 31, Part 1, No. 5a, 1514 (1992).

14. H. Schmidt-Kloiberg, G. Paltauf and E. Reichel, J. Appl. Phys., 66, 4149 (1989).

15. S. V. Egerev and A. E. Pashin, Acoustical Physics, 39, 43 (1993).

16. K. S. Shifrin, Trudy GGO, 109, 179 (1961) (in Russian).

17. J. D. Lindberg and J. B. Gillespie, Appl. Opt., 16, 2628 (1977).

18. K. A. Naugol'nykh and N. A. Roi, Electric Discharges in Water (Nauka, Moscow, 1971) (in Russian). 